\title{
Comprehensive detection of recurring genomic abnormalities: a targeted sequencing approach for multiple myeloma
}

\author{
Venkata Yellapantula', Malin Hultcrantz $\mathbb{B}^{1,2}$, Even H. Rustad (1)', Ester Wasserman ${ }^{3}$, Dory Londono ${ }^{3}$, \\ Robert Cimera ${ }^{3}$, Amanda Ciardiello ${ }^{1}$, Heather Landau ${ }^{4}$, Theresia Akhlaghi ${ }^{1}$, Sham Mailankody ${ }^{1}$, Minal Patel ${ }^{5}$, \\ Juan Santiago Medina-Martinez (i) 5 , Juan Esteban Arango Ossa ${ }^{5}$, Max Fine Levine ${ }^{5}$, Niccolo Bolli, ${ }^{6,7}$, \\ Francesco Maura ${ }^{1}$, Ahmet Dogan (10) ${ }^{8}$ Elli Papaemmanuil, ${ }^{5,9}$, Yanming Zhang ${ }^{3}$ and Ola Landgren (1)
}

\begin{abstract}
Recent genomic research efforts in multiple myeloma have revealed clinically relevant molecular subgroups beyond conventional cytogenetic classifications. Implementing these advances in clinical trial design and in routine patient care requires a new generation of molecular diagnostic tools. Here, we present a custom capture next-generation sequencing (NGS) panel designed to identify rearrangements involving the IGH locus, arm level, and focal copy number aberrations, as well as frequently mutated genes in multiple myeloma in a single assay. We sequenced 154 patients with plasma cell disorders and performed a head-to-head comparison with the results from conventional clinical assays, i.e., fluorescent in situ hybridization (FISH) and single-nucleotide polymorphism (SNP) microarray. Our custom capture NGS panel had high sensitivity (>99\%) and specificity (>99\%) for detection of IGH translocations and relevant chromosomal gains and losses in multiple myeloma. In addition, the assay was able to capture novel genomic markers associated with poor outcome such as bi-allelic events involving TP53. In summary, we show that a multiple myeloma designed custom capture NGS panel can detect IGH translocations and CNAs with very high concordance in relation to FISH and SNP microarrays and importantly captures the most relevant and recurrent somatic mutations in multiple myeloma rendering this approach highly suitable for clinical application in the modern era.
\end{abstract}

\section{Introduction}

Multiple myeloma is a heterogeneous disease in terms of genomic alterations, clinical presentation, and survival outcomes. The genetic landscape in multiple myeloma is complex and historically includes two main categories of abnormalities: hyperdiploidy, defined as gains of odd numbered chromosomes, and immunoglobulin heavy

\footnotetext{
Correspondence: Malin Hultcrantz (hultcram@mskcc.org) or Yanming Zhang (zhangy1@mskcc.org) or Ola Landgren (landgrec@mskcc.org)

${ }^{1}$ Myeloma Service, Department of Medicine, Memorial Sloan Kettering Cancer Center, New York, NY, USA

${ }^{2}$ Department of Medicine Solna, Karolinska Institute, Stockholm, Sweden Full list of author information is available at the end of the article.

These authors contributed equally: Venkata Yellapantula, Malin Hultcrantz These authors contributed equally: Ola Landgren, Yanming Zhang, Elli

Papaemmanuil
}

chain $(I G H)$ translocations, including $\mathrm{t}(4 ; 14), \mathrm{t}(6 ; 14)$, $\mathrm{t}(11 ; 14), \mathrm{t}(14 ; 16)$, and $\mathrm{t}(14 ; 20)^{1-3}$. In addition, recurrent chromosomal gains and losses have been reported, e.g. gain $1 \mathrm{q}$, del(13q), and $\operatorname{del}(17 \mathrm{p})^{2,3}$. Some of these aberrations define subgroups of patients associated with poor prognosis in the majority of published studies, e.g. $t(4 ; 14)$, $\mathrm{t}(14 ; 16)$, and $\operatorname{del}(17 \mathrm{p})^{1,4}$.

The advent of next-generation sequencing (NGS) has progressively expanded our knowledge of multiple myeloma biology identifying new and recurrent driver events such as single-nucleotide variants (SNVs) and focal deletions ${ }^{5-9}$. Emerging data suggest that the current high-risk definition can be further improved by integration of recurrent mutations and distinct cytogenetic profiles with the International Staging System ${ }^{10-14}$. 
Specifically, bi-allelic events including TP53 and more than three copies of 1q have been recently associated with poor outcomes ${ }^{5,8,15}$.

Currently, in the standard of care setting, conventional chromosome analysis, multiple myeloma targeted fluorescence in situ hybridization (FISH) panels, and singlenucleotide polymorphism (SNP) microarrays are used to detect chromosome translocations and gains and losses in multiple myeloma ${ }^{2,3}$. Conventional chromosome analysis is labor intensive, has low genomic resolution, and is often inadequate due to low mitotic activity and low percentage of plasma cells in bone marrows ${ }^{16}$. Although FISH is accurate to define distinct recurrent aberrations, it is limited to the targets of the selected probes not allowing a comprehensive cytogenetic characterization. SNP microarrays have been used to summarize copy number changes in multiple myeloma. This approach still relies on FISH for the detection of IGH translocations and usually requires about $15-20 \%$ or more aberrant plasma cell infiltration of the bone marrow which in turn limits the availability to do parallel comprehensive genomic analysis including $\mathrm{V}(\mathrm{D}) \mathrm{J}$ profiling for minimal residual disease characterization/tracking ${ }^{17,18}$. Furthermore, neither FISH nor SNP microarray approaches are able to capture somatic point mutations.

We were motivated to develop a new targeted NGS assay designed to capture frequently defined multiple myeloma subtypes. Specifically, the assay captures recurrent $I G H$ translocations, arm level, and focal copy number alterations (CNAs), as well as frequently mutated genes in multiple myeloma ur aim was to develop a novel strategy that could replace current standard of care assays and to capture mutational status in multiple myeloma in a single assay. In a head-to-head comparison with standard of care FISH and SNP microarrays, our assay revealed an extremely high concordance (sensitivity $>99 \%$ and specificity $>99 \%$ ) for both IGH translocations and CNAs. Additionally, we captured mutations and bi-allelic events relevant to clinical outcomes in multiple myeloma. Our study provides strong evidence that multiple myeloma designed custom capture NGS panels are of directly clinical relevance and can be used as a novel strategy to replace the current standard of care techniques.

\section{Methods}

\section{Custom capture next-generation sequencing panel}

In this head-to-head comparison of standard of care multiple myeloma genomic sub-typing assays (i.e. FISH and SNP microarrays) and NGS-based genomic sub-typing, we used an in-house developed multiplex custom capture NGS assay (named "myTYPE") which has been designed to detect the most common and relevant genomic aberrations in multiple myeloma ${ }^{5-9,19,20}$. To capture IGH (14q32) rearrangements, we included the canonical IGH locus. CNAs are assessed through genomewide representation of SNPs, one in every $3 \mathrm{Mb}$, to enable detection of CNAs. To capture focal events, SNPs were tiled at a higher density in loci and genes which are commonly deleted or amplified in multiple myeloma ${ }^{3}$. Additionally, we included 120 genes that were selected on the basis of (a) genes frequently mutated in multiple myeloma from earlier reports ${ }^{5-7,21-26}$, (b) genes that are involved in important signaling pathways in multiple myeloma, e.g. the MAPK and NFKB pathways (Supplementary Table $)^{6}$, (c) treatment targets and candidate genes for drug resistance in multiple myeloma (e.g., $C R B N$, IKZF1, and $I K Z F 3)^{27}$, and (d) candidate genes and SNPs associated with an increased susceptibility of developing multiple myeloma ${ }^{28}$. The total target space was $2.06 \mathrm{Mb}$. The final bait design was created using Nimblegen SeqCap.

\section{Patient cohort}

We identified 154 patients with plasma cell disorders who presented to Memorial Sloan Kettering Cancer Center (MSKCC) between 2015 and 2018 and had samples collected for conventional cytogenetic evaluation as well as samples collected under the MSKCC's institutional tissue acquisition protocol. The bone marrow aspirate specimens underwent CD138-positive enrichment through magnetic bead sorting and were divided for FISH and SNP microarray testing. DNA was extracted using commercial Qiagen DNA extraction kits and used for SNP microarray and sequencing with the custom capture NGS panel. In addition, 16 unmatched bone normal marrow samples from healthy donors were used as assay controls to filter sequencing and chemistry specific artifacts and germline variation. All patients had consented to MSKCC's institutional tissue acquisition protocol and the study was approved by the MSKCC Institutional Review Board.

\section{FISH and SNP microarray}

FISH panels for multiple myeloma included probes for $\mathrm{t}(4 ; 14), \mathrm{t}(6 ; 14), \mathrm{t}(8 ; 14), \mathrm{t}(11 ; 14), \mathrm{t}(14 ; 16)$, and $\mathrm{t}(14 ; 20)$ from Abbott Molecular, Des Plaines, IL and Metasystems, Newton, MA. All FISH testing was performed in the MSKCCs clinical Cytogenetics Laboratory. Between 100 and 500 cells, if available, were analyzed. FISH probes for translocations $t(4 ; 14)$ and $t(11 ; 14)$ were tested in all patients while in six patients, there was not enough cell material to test for $t(6 ; 14), t(8 ; 14), t(14 ; 16)$, and $t(14 ; 20)$.

SNP microarrays with 2.67 million probes including 750 thousand common and rare SNP probes (Cytoscan, Affymetrix, Santa Clara, CA) were performed following manufacturers protocol. Data analysis was performed using Affymetrix ChAS 3 software and ASCAT ${ }^{29}$. Copy neutral loss of heterozygosity $(\mathrm{CN}-\mathrm{LOH})$ was reported if 
the size was at least $10 \mathrm{Mb}$ at a terminal region or $20 \mathrm{Mb}$ for an interstitial CN-LOH. For CNA comparison in this study, only commonly recurrent multiple myeloma aberrations: $\operatorname{del}(1 p)$, gain 1q, $\operatorname{del}(6 q), \operatorname{del}(8 p), \operatorname{del}(13 q), \operatorname{del}(14 q)$, $\operatorname{del}(16 q)$, del(17p), and hyperdiploidy, were evaluated.

\section{Custom capture NGS panel data generation and processing}

After DNA extraction, barcoded sequence libraries (New England Biolabs, Kapa Biosystems, Wilmington, MA, USA) were subjected to capture by hybridization (Nimblegen SeqCap, Madison, WI, USA). Between 100 and $200 \mathrm{ng}$ of gDNA was used as input for library construction. DNA was subsequently sequenced on an Illumina HiSeq 2500 sequencer to generate paired-end 101bp reads.

Translocations were analyzed using the algorithms BRASS and Delly ${ }^{30,31}$. CNVKit and FACETS were used to identify somatic CNAs ${ }^{32,33}$. Together with results from CNVKit, B-Allele frequencies using SNPs from the 1000 genomes project were used to identify regions of $\mathrm{CN}$ $\mathrm{LOH}$. The 16 unmatched control samples were combined into a pooled reference for CNA comparison. SNVs and indels were called combing results from CaVEMan, Strelka2, Mutect2, and Pindel ${ }^{34-37}$. Bi-allelic inactivation was assessed in patients with multiple myeloma and was defined as having a CNA, e.g. arm level or focal deletion, and somatic mutation affecting the same gene. Tumor-specific $I G H-\mathrm{V}(\mathrm{D}) \mathrm{J}$ rearrangement sequences were identified using the MiXCR algorithm $^{38,39}$. Detailed information on variant calling is given in the Supplementary Material.

\section{Results}

\section{Patient cohort}

Bone marrow aspirates from 154 patients with plasma cell disorders; multiple myeloma $(N=118)$, smoldering multiple myeloma $(N=17)$, monoclonal gammopathy of undetermined significance) $(N=5)$, plasma cell leukemia $(N=1)$, and light-chain (AL) amyloidosis $(N=13)$ were included in the study (Table 1). With our custom capture NGS assay (myTYPE), IGH translocations were detected in $51 \%$, multiple myeloma relevant CNAs in $83 \%$, and somatic mutations in $85 \%$ percent of patients (Fig. 1).

\section{Assay coverage and sample purity}

Sequencing using the custom capture NGS assay generated a median of 38 million paired-end reads per sample resulting in a median target coverage of $651 \mathrm{x}$ per sample (Supplementary Fig. S1). The coverage across the IGH locus, exonic regions, genome-wide copy number SNPs, and finger printing SNPs were homogeneous (Supplementary Fig. S2).
Table 1 Patients' characteristics.

\begin{tabular}{lc}
\hline Total, $\boldsymbol{N}$ (\%) & $\mathbf{1 5 4}(\mathbf{1 0 0 \% )}$ \\
\hline Median age, years (range) & $63(35-85)$ \\
Male sex, N (\%) & $76(49 \%)$ \\
Diagnosis & \\
$\quad$ Multiple myeloma, N (\%) & $118(76 \%)$ \\
Smoldering multiple myeloma, N (\%) & $17(11 \%)$ \\
Monoclonal gammopathy of undetermined & $5(3 \%)$ \\
significance, N (\%) & $1(1 \%)$ \\
Plasma cell leukemia, N (\%) & $13(8 \%)$ \\
\hline AL amyloidosis, N (\%)
\end{tabular}

The sample purity for SNP microarray was estimated using ASCAT and for the custom capture NGS assay using algorithms based on mutation calls. Median purity was $75 \%$ using ASCAT. There was a high concordance between the sample purity for the two methods (Pearson's $r^{2}=0.61$ ) (Fig. 2).

\section{Comparison myeloma targeted FISH versus the custom capture NGS assay}

Across the 154 samples that were assessed with multiple myeloma targeted FISH and our custom capture NGS assay, $78 I G H$ translocations were detected by both approaches with a $100 \%$ concordance for $t(4 ; 14), t(6 ; 14)$, $\mathrm{t}(11 ; 14), \mathrm{t}(14 ; 16)$, and $(14 ; 20)$ (Table 2). One patient had both $t(11 ; 14)$ and $t(8 ; 14)$ translocation detected using the custom capture NGS assay, only the $t(11 ; 14)$ translocation was detected with FISH in this sample (Supplementary Fig. S3). In two patients, $t(8 ; 14)$ detected by FISH was not confirmed by our custom capture NGS assay. This may be indicative of variable $I G H$ breakpoints in $M Y C$ translocations, which may be outside of the custom capture NGS assay's primer coverage (Supplementary Fig. S4). The concordance was $96 \%(N=75)$ with a sensitivity and specificity of $97 \%$ and $100 \%$, respectively, for the custom capture NGS assay using FISH as the reference. The distribution and concordance of each of the translocations in the study cohort is given in Table 2 and Figs. 3 and 4 .

\section{Comparison of SNP microarray versus the custom capture NGS assay}

Focusing on the CNAs of relevance for multiple myeloma: del(1p), gain 1q, $\operatorname{del}(6 q), \operatorname{del}(8 p), \operatorname{del}(13 q), \operatorname{del}(14 q)$, $\operatorname{del}(16 q), \operatorname{del}(17 p)$, and hyperdiploidy (HRD), there were 384 CNAs identified by both assays, 3 uniquely identified by SNP microarray and 15 uniquely identified by the custom capture NGS assay (Figs. 2 and 3). HRD, defined as extra copies of two or more of the odd chromosomes, was detected in 73 patients with $100 \%$ concordance 

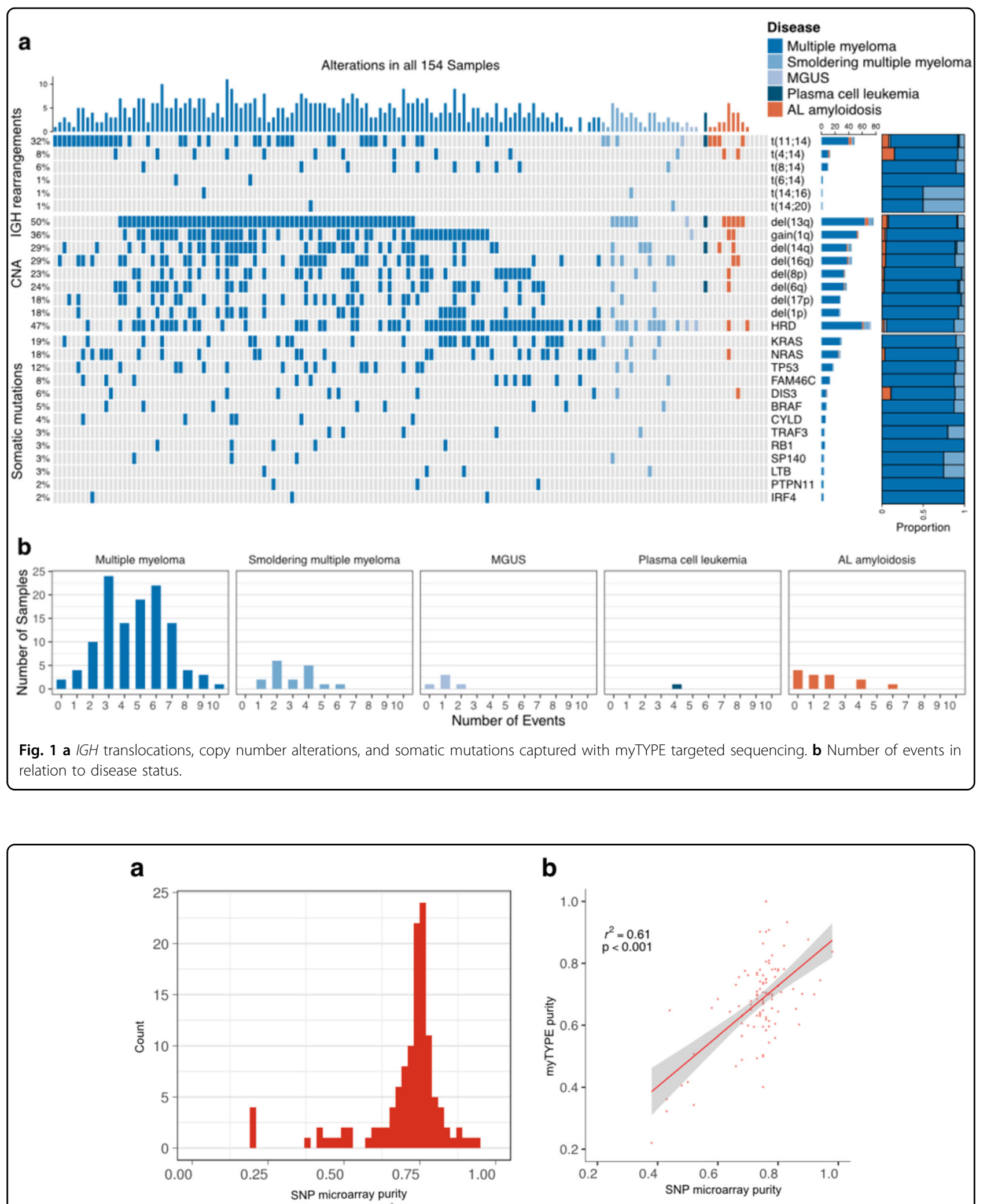

Fig. 2 a Sample purity estimates SNP microarray and $\mathbf{b}$ correlation between sample purity estimated by SNP microarray and myTYPE sequencing. 
Table 2 Common genomic aberrations and their detection rates using the custom capture next-generation sequencing (NGS) assay and fluorescent in situ hybridization (FISH) and single-nucleotide polymorphism (SNP) microarray, respectively.

\begin{tabular}{|c|c|c|c|c|c|}
\hline Aberration & $\begin{array}{l}\text { FISH/SNP microarray } \\
\text { and NGS assay }\end{array}$ & FISH/SNP microarray Unique & NGS assay Unique & Sensitivity, $\%\left(95 \% \mathrm{Cl}^{*}\right)$ & Specificity, $\%\left(95 \% \mathrm{Cl}^{*}\right)$ \\
\hline Overall & 483 & 6 & 15 & $0.99(0.97-1)$ & $0.99(0.99-1)$ \\
\hline $\operatorname{Del}(1 p)$ & 28 & 1 & 0 & $0.97(0.82-1)$ & $1(0.97-1)$ \\
\hline Gain(1q) & 55 & 1 & 0 & $0.98(0.9-1)$ & $1(0.96-1)$ \\
\hline $\operatorname{Del}(6 q)$ & 35 & 1 & 2 & $0.97(0.85-1)$ & $0.98(0.94-1)$ \\
\hline $\operatorname{Del}(8 p)$ & 33 & 0 & 2 & $1(0.89-1)$ & $0.98(0.94-1)$ \\
\hline $\operatorname{Del}(13 q)$ & 77 & 1 & 0 & $0.99(0.93-1)$ & $1(0.95-1)$ \\
\hline $\operatorname{Del}(14 q)$ & 40 & 0 & 5 & $1(0.91-1)$ & $0.96(0.9-0.99)$ \\
\hline $\operatorname{Del}(16 q)$ & 42 & 0 & 3 & $1(0.92-1)$ & $0.97(0.93-0.99)$ \\
\hline $\operatorname{Del}(17 p)$ & 26 & 0 & 2 & $1(0.87-1)$ & $0.98(0.95-1)$ \\
\hline HRD & 73 & 0 & 0 & $1(0.95-1)$ & $1(0.96-1)$ \\
\hline $\mathrm{t}(4 ; 14)$ & 13 & 0 & 0 & $1(0.75-1)$ & $1(0.97-1)$ \\
\hline $\mathrm{t}(6 ; 14)$ & 1 & 0 & 0 & $1(0.03-1)$ & $1(0.98-1)$ \\
\hline $\mathrm{t}(8 ; 14)$ & 8 & 2 & 1 & $0.8(0.44-0.97)$ & $0.99(0.96-1)$ \\
\hline$t(11 ; 14)$ & 48 & 0 & 0 & $1(0.93-1)$ & $1(0.97-1)$ \\
\hline$t(14 ; 16)$ & 2 & 0 & 0 & $1(0.16-1)$ & $1(0.98-1)$ \\
\hline$t(14 ; 20)$ & 2 & 0 & 0 & $1(0.16-1)$ & $1(0.98-1)$ \\
\hline
\end{tabular}

FISH fluorescent in situ hybridization, SNP single-nucleotide polymorphism, NGS next-generation sequencing, $C I$ confidence interval, $H R D$ hyperdiploidy

between SNP microarray and our custom capture NGS assay. Del(13q) was the most frequent CNA (50\%), followed by gain 1q (36\%) (Table 2). Del(17p) was found in 26 patients (17\%) by both SNP microarray and the custom capture NGS assay while two additional arm-level 17p deletions were detected uniquely by the NGS assay.

The overall sensitivity and specificity were $99 \%$ and $99 \%$, respectively, for our custom capture NGS assay using SNP microarray as reference. Copy neutral LOH was detected using both SNP microarrays and the custom capture NGS assay (CNAs and copy neutral LOH are shown in Supplementary Figs. S5-S7). In addition, of the 55 cases where a 1q gain was identified, more than one extra copy of 1q was detected in eight cases (Supplementary Fig. S8). There was no correlation between sample purity and detection of genomic events in this study, similar to what was observed in CoMMpass and previous whole-genome sequencing studies ${ }^{40,41}$. There were no samples that did not harbor any genomic aberrations; the less common aberrations are specified in the Supplementary Material.

\section{Mutations, small insertions, and deletions by the custom capture NGS assay}

At least one non-synonymous mutation was identified in 132 of 154 (85\%) samples. In these 132 samples, a total of 362 non-synonymous SNVs and 51 Indels were detected by our custom capture NGS assay (median 3/ sample). We found that $19 \%$ and $18 \%$ of the samples each harbored KRAS and NRAS mutations, respectively, and $5 \%$ of patients harbored a BRAF V600E mutation. TP53, FAM $46 C$, and DIS3 were detected in $12 \%, 8 \%$, and $6 \%$ of patients, respectively (Fig. 1, Supplementary Figs. S9 and S10). Moreover, we identified a tumor-specific clonal V (D)J rearrangement sequence in 127 of 154 samples (82\%).

With the availability of arm level and focal CNA assessment and mutational data using our custom capture NGS assay, we identified 20 patients with bi-allelic events affecting TP53, FAM46C, RB1, and TRAF3 (Fig. 5). Specifically, among patients with a $17 \mathrm{p}$ deletion $(N=29), 13$ patients (45\%) had mutation of the non-deleted allele causing bi-allelic inactivation of TP53 (Fig. 5).

\section{Discussion}

This is the first large-scale head-to-head comparison of standard of care targeted FISH panel and SNP microarray versus a multiple myeloma designed custom capture NGS assay designed to replace current standard of care prognostic bone marrow assays for multiple myeloma patients with a single test. The study is based on bone marrow specimens from a well-defined cohort of 154 patients with 

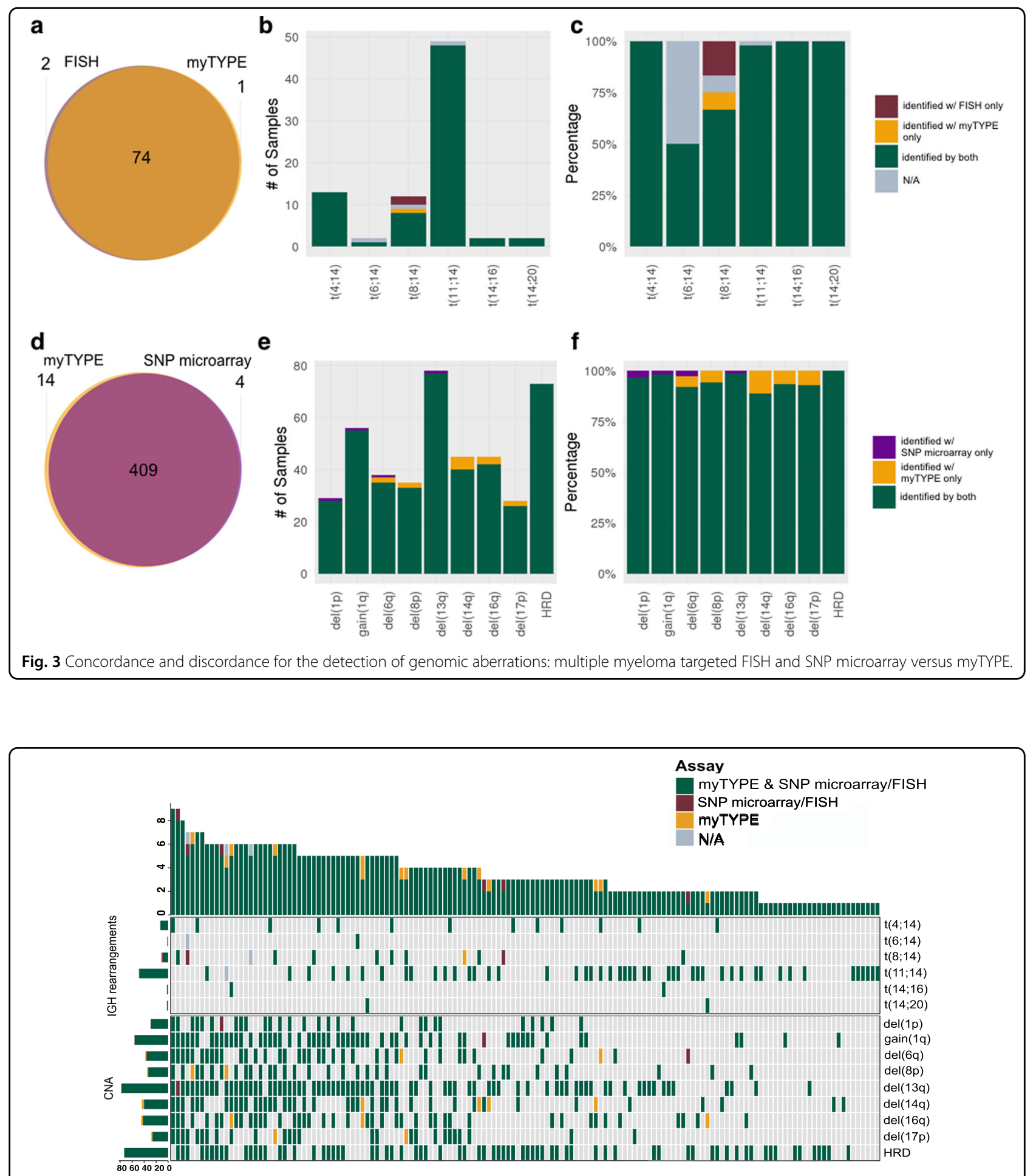

Fig. 4 Comparison of multiple myeloma targeted FISH and SNP microarray versus myTYPE.

plasma cell disorders. Overall, we found an extremely high concordance (sensitivity $>99 \%$ and specificity $>99 \%$ ) between our custom capture NGS assay and conventional multiple myeloma targeted FISH/SNP microarrays for detection of $I G H$ translocations and CNAs. In addition to profiling of $I G H$ translocations and CNAs, our custom capture NGS assay captures relevant somatic mutations in multiple myeloma as well as bi-allelic inactivations that are critically relevant for prognostic assessment. Our findings are of high clinical importance as they support the 


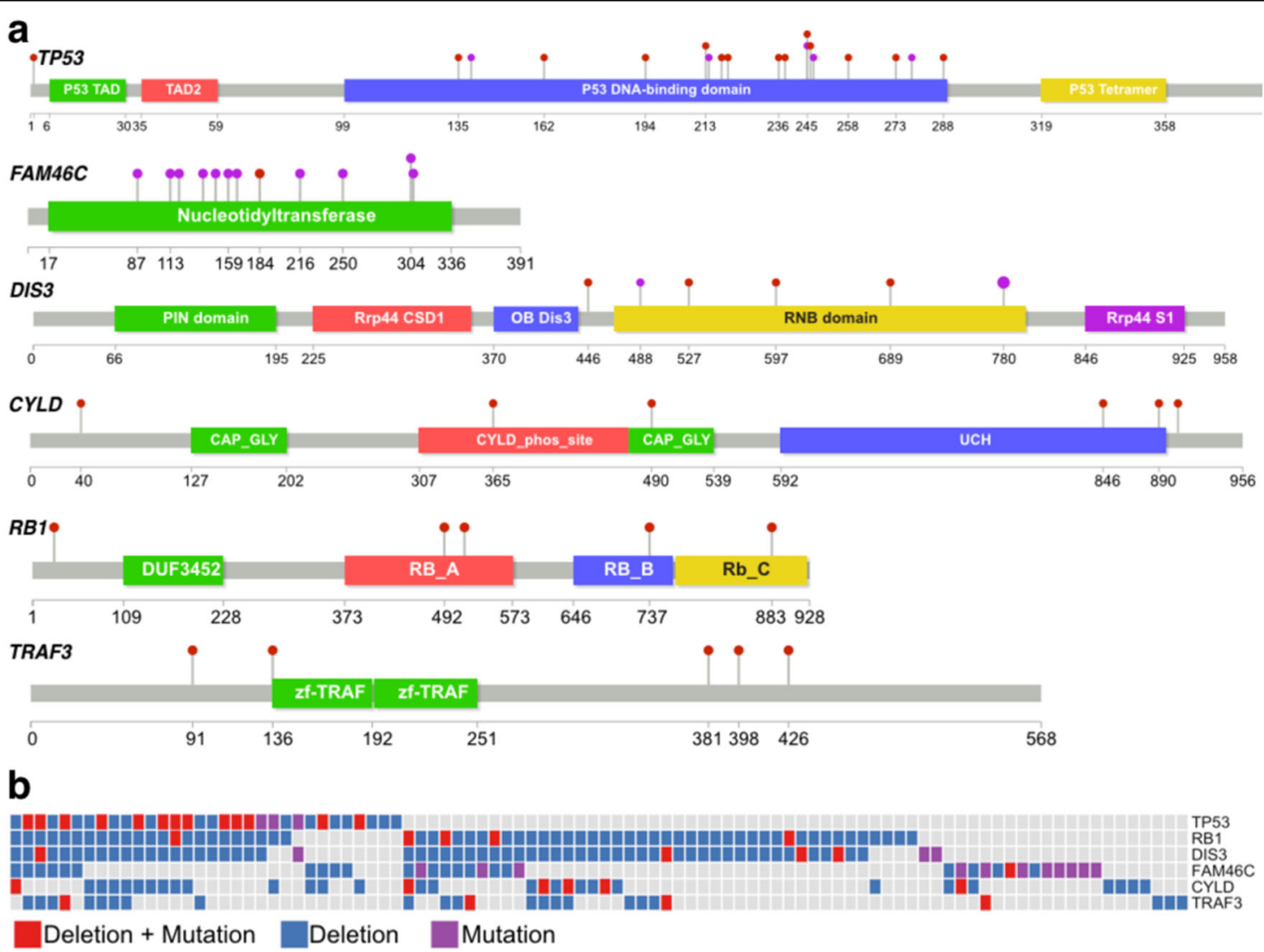

Fig. 5 a Distribution of somatic mutations in selected tumor suppressor genes. b Bi-allelic events detected through sequencing with myTYPE.

use of one single assay that can replace current standard of care bone marrow assays for multiple myeloma.

For any new assay that is introduced in clinical practice, it should have higher sensitivity and specificity or be more efficient than current options. Multiple myeloma targeted FISH and SNP microarray are labor intensive, require relatively large amounts of bone marrow material, and have limited sensitivity. In this head-to-head comparison, our custom capture NGS assay had a very high sensitivity and specificity in comparison with FISH and SNP microarray in capturing the recurrent genomic aberrations in multiple myeloma. The concordance for the common IGH translocations and HRD was $100 \%$. The only translocation that was not fully captured using the custom capture NGS assay was $t(8 ; 14)$ likely due variable $I G H$ breakpoints in MYC translocations which may partly be outside of the NGS assay primer coverage ${ }^{42}$. Regarding CNAs, our custom capture NGS assay identified a number of additional alterations not detected through SNP microarray analyses.

As for capturing somatic mutations, available NGS panels, such as MSK-IMPACT and FoundationOne CDx, have proven their utility in identifying mutations and $\mathrm{CNAs}^{43,44}$. However, none of these NGS panels have been designed to capture $I G H$ translocations relevant to multiple myeloma. Because approximately $50 \%$ of all patients with multiple myeloma have $I G H$ translocations, multiple myeloma targeted FISH and SNP microarrays are still required in parallel with these NGS panels ${ }^{43,44}$. Going forward, it seems logical to conjecture that other groups with interest and expertise in genomics and bioinformatics will develop multiple myeloma designed custom capture NGS assays for clinical use in the future. Also, as new scientific insights become available, there will be need for updated versions of existing multiple myeloma designed custom capture NGS assays.

Analysis of somatic mutations in multiple myeloma is becoming increasingly important for better prognostic assessments and to identify actionable mutations for targeted therapy. In the current analysis, we found that the prevalence of KRAS and NRAS as well as the overall landscape of somatic mutations were in line with the published literature ${ }^{5-7}$. We conclude that our custom capture NGS assay precisely captures comprehensive genomic abnormalities beyond FISH and SNP microarrays and shows that it is possible to replace current standard of care prognostic bone marrow assays for multiple myeloma patients with a single test. 
High-risk multiple myeloma is a relative terminology that is subject to change as modern effective therapies are constantly being improved ${ }^{10-12}$. Per the International Myeloma Working Group consensus criteria, IGH translocations $\mathrm{t}(4 ; 14), \mathrm{t}(14 ; 16)$, and $17 \mathrm{p}$ deletions are defined as high-risk multiple myeloma and 1q gains have been included in earlier versions of high-risk definitions $^{4,45}$. More recently, novel potential oncogenes and tumor suppressor genes have been reported in multiple myeloma, e.g. PTPN11, PRKD2, IDH1/2, HUWE1, and $U B R 5$ in addition to the known driver genes in multiple myeloma, i.e. KRAS, NRAS, BRAF, TP53, FAM46C, DIS3, and more ${ }^{8}$. Importantly, bi-allelic inactivation of tumor suppressor genes, particularly TP53, have been linked to a dismal outcome in multiple myeloma ${ }^{15}$. As illustrated in this study, an advantage of our custom capture NGS assay is the integrated capture of CNAs and mutations co-occurring in the same genes, allowing for the assessment of bi-allelic events involving different types of aberrations. Indeed, in 29 multiple myeloma patients with 17p deletions, our custom capture NGS assay detected TP53 mutations in 13 patients (45\%), resulting in bi-allelic TP53 inactivation. There is an urgent need for functional studies designed to better understand underlying mechanisms of such cases, which, in turn will allow development of more rational therapies for these patients.

Strengths of this study are the head-to-head comparison of a large patient cohort, and as shown consistently throughout this study, there is high concordance between conventional assays (multiple myeloma targeted FISH panels and SNP microarrays) and our custom capture NGS assay when it comes to detection of IGH translocations and CNAs. Limitations include the lack of normal samples for comparison of mutation calling and it imposes challenges for detection of $\mathrm{LOH}$ as the inference is based on tumor variant allele frequencies alone. Furthermore, our custom capture NGS assay is designed to capture the IGH locus where $>95 \%$ of IGH translocations occur which may have impacted the detection of $\mathrm{t}(8 ; 14)$ translocations in this study. Also, immunoglobulin lightchain translocations, i.e. IGK and IGL, may be involved in the pathogenesis in multiple myeloma, however, due to the scarcity of knowledge on their impact, our custom capture NGS assay was not designed to detect IGK and $I G L$ translocations. Future updated versions of our assay will be revised/expanded in its capture based on biological discoveries.

In conclusion, our multiple myeloma developed custom capture NGS assay captures IGH translocations, CNAs, and relevant somatic mutations and thus enables translation of information from recent large sequencing efforts into clinical care. The large head-to-head comparison between targeted FISH panels and SNP microarrays versus our custom capture NGS assay revealed extremely high concordance in regard to detection of relevant $I G H$ translocations and CNAs in multiple myeloma. The ability to capture relevant somatic mutations as well as recurrent $I G H$ translocations and all CNAs with high sensitivity and specificity in a single assay further supports the clinical strengths of multiple myeloma developed custom capture NGS assays as optimal for patient care in the modern era.

\section{Acknowledgements}

Funding support for this publication was provided by the Multiple Myeloma Research Foundation (MMRF), the Swedish Research Council, and the Memorial Sloan Kettering Core Grant (P30 CA008748).

\section{Author details}

${ }^{1}$ Myeloma Service, Department of Medicine, Memorial Sloan Kettering Cancer Center, New York, NY, USA. ²Department of Medicine Solna, Karolinska Institute, Stockholm, Sweden. ${ }^{3}$ Cytogenetics Laboratory, Department of Pathology, Memorial Sloan Kettering Cancer Center, New York, NY, USA. ${ }^{4}$ Bone Marrow Transplant Service, Department of Medicine, Memorial Sloan Kettering Cancer Center, New York, NY, USA. ${ }^{5}$ Center for Hematological Malignancies, Department of Medicine, Memorial Sloan Kettering Cancer Center, New York, NY, USA. ${ }^{6}$ University of Milan, Department of Oncology and Onco-Hematology, Milan, Italy. ${ }^{7}$ Fondazione IRCCS Istituto Nazionale dei Tumori, Department of Medical Oncology and Hematology, Milan, Italy. ${ }^{8}$ Hematopathology Laboratory, Department of Pathology, Memorial Sloan Kettering Cancer Center, New York, NY, USA. ' Epidemiology \& Biostatistics, Department of Medicine, Memorial Sloan Kettering Cancer Center, New York, NY, USA

\section{Author contributions}

V.Y., M.H., E.P., Y.Z., and O.L. designed the study; V.Y., M.H., E.H.R., Y.Z., E.P., F.M., and O.L. analyzed and interpreted the data; M.H., V.Y., and O.L. wrote the paper. All authors provided critical input on the results and gave final approval of the manuscript.

\section{Conflict of interest}

M.H. has received funding from the Multiple Myeloma Research Foundation, the Swedish Research Council, the Swedish Cancer Society, Karolinska Institute Foundations, and the Swedish Blood Cancer Foundation. N.B. has received Honoraria/ad boards from: Celgene, Janssen, Novartis. E.H. is a Josie Robertson Investigator, an EHA Fellow, and an ASH Scholar. O.L. has received research funding from: National Institutes of Health (NIH), U.S. Food and Drug Administration (FDA), Multiple Myeloma Research Foundation (MMRF), International Myeloma Foundation (IMF), Leukemia and Lymphoma Society (LLS), Perelman Family Foundation, Rising Tides Foundation, Amgen, Celgene, Janssen, Takeda, Glenmark, Seattle Genetics, Karyopharm; Honoraria/ad boards: Adaptive, Amgen, Binding Site, BMS, Celgene, Cellectis, Glenmark, Janssen, Juno, Pfizer; and serves on Independent Data Monitoring Committees (IDMCs) for clinical trials lead by Takeda, Merck, Janssen, and Theradex. There is a pending patent application regarding the myTYPE assay (M.H., E.P., and O.L.). The remaining authors declare no relevant conflicts of interest.

\section{Publisher's note}

Springer Nature remains neutral with regard to jurisdictional claims in published maps and institutional affiliations.

Supplementary Information accompanies this paper at (https:/doi.org/ 10.1038/s41408-019-0264-y).

Received: 30 August 2019 Revised: 31 October 2019 Accepted: 15 November 2019

Published online: 11 December 2019 


\section{References}

1. Manier, S., Salem, K., Glavey, S. V., Roccaro, A. M. \& Ghobrial, I. M. Genomic aberrations in multiple myeloma. Cancer Treat. Res. 169, 23-34 (2016).

2. Robiou du Pont, S. et al. Genomics of multiple myeloma. J. Clin. Oncol. 35 963-967 (2017)

3. Manier, S. et al. Genomic complexity of multiple myeloma and its clinical implications. Nat. Rev. Clin. Oncol. 14, 100-113 (2017).

4. Palumbo, A. et al. Revised international staging system for multiple myeloma: a report from International Myeloma Working Group. J. Clin. Oncol. 33, 2863 (2015).

5. Bolli, N. et al. Analysis of the genomic landscape of multiple myeloma highlights novel prognostic markers and disease subgroups. Leukemia $\mathbf{3 2}$ 2604-2616 (2018)

6. Lohr, J. G. et al. Widespread genetic heterogeneity in multiple myeloma: implications for targeted therapy. Cancer Cell 25, 91-101 (2014).

7. Walker, B. A. et al. Mutational spectrum, copy number changes, and outcome: results of a sequencing study of patients with newly diagnosed myeloma. J. Clin. Oncol. 33, 3911-3920 (2015)

8. Walker, B. A. et al. Identification of novel mutational drivers reveals oncogene dependencies in multiple myeloma. Blood 132, 587-597 (2018).

9. Bolli, N. et al. A DNA target-enrichment approach to detect mutations, copy number changes and immunoglobulin translocations in multiple myeloma. Blood Cancer J. 6, e467 (2016).

10. Jakubowiak, A. J. et al. A phase $1 / 2$ study of carfilzomib in combination with lenalidomide and low-dose dexamethasone as a frontline treatment for multiple myeloma. Blood 120, 1801-1809 (2012).

11. Kazandjian, D. et al. Remission and progression-free survival in patients with newly diagnosed multiple myeloma treated with carfilzomib, lenalidomide, and dexamethasone: five-year follow-up of a phase 2 clinical trial. JAMA Oncol. 4. 1781-1783 (2018)

12. Korde, N. et al. Treatment with carfilzomib-lenalidomide-dexamethasone with lenalidomide extension in patients with smoldering or newly diagnosed multiple myeloma. JAMA Oncol. 1, 746-754 (2015).

13. Greipp, P. R. et al. International staging system for multiple myeloma. J. Clin. Oncol. 23, 3412-3420 (2005).

14. Palumbo, A. et al. Revised international staging system for multiple myeloma: a report from International Myeloma Working Group. J. Clin. Oncol. 33, 2863-2869 (2015).

15. Walker, B. A. et al. A high-risk, double-hit, group of newly diagnosed myeloma identified by genomic analysis. Leukemia 33, 159-170 (2019).

16. Rajan, A. M. \& Rajkumar, S. V. Interpretation of cytogenetic results in multiple myeloma for clinical practice. Blood Cancer J. 5, e365 (2015).

17. Rustad, E. H. et al. Baseline identification of clonal V(D)J sequences for DNAbased minimal residual disease detection in multiple myeloma. PLOS ONE 14 e0211600 (2019).

18. Landgren, O. \& Rustad, E. H. Meeting report: advances in minimal residual disease testing in multiple myeloma 2018. Adv. Cell Gene Ther. 2, e26 (2019).

19. Walker, B. A. et al. Characterization of IGH locus breakpoints in multiple myeloma indicates a subset of translocations appear to occur in pregerminal center B cells. Blood 121, 3413-3419 (2013).

20. Kazandjian, D. et al. Molecular underpinnings of clinical disparity patterns in African American vs. Caucasian American multiple myeloma patients. Blood Cancer J. 9, 15 (2019).

21. Kortuem, K. M. et al. Panel sequencing for clinically oriented variant screening and copy number detection in 142 untreated multiple myeloma patients. Blood Cancer J. 6, e397 (2016).
22. Liwing, J. et al. Improved survival in myeloma patients: starting to close in on the gap between elderly patients and a matched normal population. Br. J. Haematol. 164, 684-693 (2014).

23. Christofferson, A. et al. Integrative analysis of the genomic landscape underlying multiple myeloma at diagnosis: an mmrf commpass analysis. Blood 130 , 326-326 (2017).

24. Lonial, S. et al. Interim analysis of the Mmrf Commpass Trial: identification of novel rearrangements potentially associated with disease initiation and progression. Blood 124, 722-722 (2014).

25. Rustad, E. H. et al. BRAF V600E mutation in early-stage multiple myeloma: good response to broad acting drugs and no relation to prognosis. Blood Cancer J. 5, e299 (2015)

26. Rustad, E. H. et al. Monitoring multiple myeloma by quantification of recurrent mutations in serum. Haematologica 102, 1266-1272 (2017).

27. Kortum, K. M. et al. Targeted sequencing of refractory myeloma reveals a high incidence of mutations in CRBN and Ras pathway genes. Blood $\mathbf{1 2 8}$ 1226-1233 (2016).

28. Morgan, G. J. et al. Inherited genetic susceptibility to multiple myeloma Leukemia 28, 518-524 (2014).

29. Van Loo, P. et al. Allele-specific copy number analysis of tumors. Proc. Natl Acad. Sci. USA 107, 16910-16915 (2010).

30. Nik-Zainal, S. et al. Landscape of somatic mutations in 560 breast cancer whole-genome sequences. Nature 534, 47-54 (2016).

31. Rausch, T. et al. DELLY: structural variant discovery by integrated paired-end and split-read analysis. Bioinformatics 28, i333-i339 (2012).

32. Talevich, E., Shain, A. H., Botton, T. \& Bastian, B. C. CNVkit: genome-wide copy number detection and visualization from targeted DNA sequencing. PLOS Comput. Biol. 12, e1004873 (2016).

33. Shen, R. \& Seshan, V. E. FACETS: allele-specific copy number and clonal heterogeneity analysis tool for high-throughput DNA sequencing. Nucleic Acids Res. 44, e131 (2016).

34. Raine, K. M. et al. cgpPindel: identifying somatically acquired insertion and deletion events from paired end sequencing. Curr. Protoc. Bioinformatics $\mathbf{5 2}$ 15.17.11-15.17.12 (2015)

35. Jones, D et al cgpCaVEManWrapper: simple execution of CaVEMan in order to detect somatic single nucleotide variants in NGS data. Curr. Protoc. Bioinformatics 56, 15.10.11-15.10.18 (2016).

36. Kim, S. et al. Strelka2: fast and accurate variant calling for clinical sequencing applications. Nat. Methods 15, 591-594 (2018).

37. Cibulskis, K. et al. Sensitive detection of somatic point mutations in impure and heterogeneous cancer samples. Nat. Biotechnol. 31, 213-219 (2013).

38. Bolotin, D. A. et al. MiXCR: software for comprehensive adaptive immunity profiling. Nat. Methods 12, 380-381 (2015).

39. Rustad, E. H. et al. Stability and uniqueness of clonal immunoglobulin CDR3 sequences for MRD tracking in multiple myeloma. Am. J. Hematol. 94 1364-1373 (2019).

40. Maura, F. et al. Genomic landscape and chronological reconstruction of driver events in multiple myeloma. Nat. Commun. 10, 3835 (2019).

41. Rustad E. et al. Timing the initiation of multiple myeloma. Sneak Peek. Pre-print at https://doi.org//10.2139/ssrn3409453 (2019).

42. Barwick, B. G. et al. Multiple myeloma immunoglobulin lambda translocations portend poor prognosis. Nature communications 10, 1911 (2019).

43. Zehir, A. et al. Mutational landscape of metastatic cancer revealed from prospective clinical sequencing of 10,000 patients. Nat Med. 23, 703-713 (2017)

44. He, J. et al. Integrated genomic DNA/RNA profiling of hematologic malignancies in the clinical setting. Blood 127, 3004-3014 (2016).

45. Chng, W. J. et al. IMWG consensus on risk stratification in multiple myeloma. Leukemia 28, 269-277 (2014). 